\title{
Non-linear dielectric effect in the isotropic phase above the isotropic-cholesteric phase transition
}

\author{
Prabir K. Mukherjee ${ }^{1 \ddagger}$, Sumanta Chakraborty ${ }^{1}$ and Sylwester J. Rzoska ${ }^{2,3}$ \\ ${ }^{1}$ Department of Physics, Presidency University, 86/1 College Street, Kolkata - 700 073, India \\ 2 Institute of Physics, Silesian University, Uniwersytecka 4, 40-007 Katowice, Poland \\ ${ }^{3}$ Institute of High Pressure Physics PAS, ul. Sokolowska 27/39, 00-143 Warsaw, Poland
}

(Dated: November 5, 2018)

\begin{abstract}
Using the Landau-de Gennes theory, the temperature, pressure and frequency dependence of the non-linear effect in the isotropic phase above the isotropic-cholesteric phase transition is calculated. The influence of pressure on the isotropic-cholesteric phase transition is discussed by varying the coupling between the orientational order parameter and the macroscopic polarization of polar cholesterics. Comparing the results of the calculations with existing data, we finally conclude that the model provides a description of the isotropic-cholesteric transition that takes all experimentally known features of the unusual negative and positive pretransitional effect in the isotropic phase of the system into account in a qualitatively correct way.
\end{abstract}

Keywords: Non-linear dielectric effect, Liquid crystals, Phase transition

\footnotetext{
$\ddagger$ Corresponding author: Tel: +913322411977, E-mail address: pkmuk1966@gmail.com
} 


\section{INTRODUCTION}

The cholesteric $(\mathrm{Ch})$ mesophase is locally very similar to the nematic $(\mathrm{N})$ phase except that it is composed of optically active molecules. When optically active material form a nematic phase, the preferred direction of the long-molecular axis varies in a direction throughout the medium in a regular way and displays a continuous twist along the optic axis leading to a helical structure. Thus the cholesteric phase is a distorted nematic phase.

While the research on the isotropic-nematic (I-N) phase transition has been going on over the past few decades, there is also increasing interest in the isotropic-cholesteric (I-Ch) transition [112]. De Gennes [1] studied the pretransitional effect in the isotropic phase of cholesterics. He pointed out that the pretransitional effect in the isotropic phase emerges due to the presence of chiral aggregates. The I-Ch transition is believed to be first order [2 -5]. In the isotropic phase of cholesterics the chirality exists only over a short temperature range [2]. Brazovskii et al [7, 8] predict a first order transition to the spiral phase, occurring in a region of substantial manifestation of critical anomalies due to the effect of critical fluctuations. Longa et al [10] studied the phase diagrams of cholesteric liquid crystal with a generalized Landau-de Gennes theory. Muralidhar et al [11] measured the ultrasonic absorption both in the cholesteric and isotropic phases as a function of both temperature and frequency. They observed the anomalous behavior of the absorption in both the phases. Seidin et al [12] studied the mean field phase diagram of a bulk cholesteric liquid crystal subjected to an externally applied field.

The quantitative characteristic of the I-Ch transition delivered a series of linear and nonlinear dielectric permittivity investigations in chiral isopentylcyanobiphenyl $\left(5^{*} \mathrm{CB}\right)$ carried out by Rzoska and co-workers [13-15]. They measured the pressure dependence of the non-linear dielectric effect $(N D E)$ in the isotropic phase. It was found that $N D E$ decreased strongly towards negative values on pressuring. This unusual behavior is opposite to the I-N and isotropic-smectic-A (ISmA) transitions [16 20]. Further it was observed that for the lower measurement frequency the $N D E$ strongly increases in the direction of positive values on approaching the I-Ch transition. For higher measurement frequencies the negative-sign pretransitional anomaly in the isotropic phase occurs. The clear evidence for the pretransitional behavior of the $N D E$ in the isotropic phase of the I-Ch transition was observed. Thus the I-Ch phase transition is associated with a pronounced pretransitional $N D E$ since the aligning electric field $\mathbf{E}$ couples to the critical fluctuations. The pressure study on the $N D E$ suggests that the same critical-like behavior with exponent $\alpha=0.5$ occurs for the I-Ch transition similar to the I-N and I-SmA transitions. 
In spite of these experimental and theoretical efforts, there remains still number of key questions concerning the properties of the I-Ch transition. In particular, the natural supercooling of the isotropic phase and the anomalies in the temperature and pressure dependence of the precritical scattering of light and the $N D E$ require deeper investigation. It is worthwhile here to point out that the Landau theories described above are able to explain some of the main experimental observations at ambient pressure. There is no such theoretical study on the pressure effect on the I-Ch transition.

The purpose of the present paper is to study the pressure effect on the I-Ch transition. We calculate the pressure and frequency dependence of the $N D E$ in the isotropic phase above the I-Ch phase transition. The model gives a correct description of the qualitative nature of the positive and negative pretransitional behavior of the $N D E$ in the isotropic phase of the I-Ch transition.

\section{THEORY}

In this section we consider the Landau-de Gennes theory of cholesteric liquid crystals. The cholesteric order parameter proposed by de Gennes [1, 21] is a symmetric, traceless tensor described by $Q_{i j}=\frac{S}{2}\left(3 n_{i} n_{j}-\delta_{i j}\right)$. Here $\mathbf{n}=\left(n_{x}, n_{y}, n_{z}\right)$ and $S$ is the magnitude of the nematic order parameter. In the geometry which is considered here the cholesteric director is assumed to be $n_{i}=e_{x} \cos k_{0} z+e_{y} \sin k_{0} z$. Here the direction of the helix axis is in the $z$ direction. Thus the structure of the cholesteric liquid crystal is periodic with a spatial period given by $L=\pi /\left|k_{0}\right|$. The unique feature of $5^{*} \mathrm{CB}$ is that its structure resembles one of the classical liquid crystalline compounds $5 \mathrm{CB}$. The compound $5^{*} \mathrm{CB}$ composed of strongly polar molecules. The external electric field induces a macroscopic polarization $\mathbf{P}_{\mathbf{m}}$. Then the free energy of the polar cholesteric phase is a function of the orientational order parameter $Q_{i j}$ and the polarization $\mathbf{P}_{\mathbf{m}}$. Taking into account the relatively small value of the induced polarization, one can expand the free energy in powers of $Q_{i j}$ and $\mathbf{P}_{\mathbf{m}}$

$$
\begin{aligned}
F= & F_{0}+\frac{1}{2} A Q_{i j} Q_{i j}-\frac{1}{3} B Q_{i j} Q_{j k} Q_{k i} \\
& +\frac{1}{4} C_{1}\left(Q_{i j} Q_{i j}\right)^{2}+\frac{1}{4} C_{2} Q_{i j} Q_{j k} Q_{k l} Q_{l i} \\
& +\frac{1}{2} L_{1} \nabla_{i} Q_{j k} \nabla_{i} Q_{j k}+\frac{1}{2} L_{2} \nabla_{i} Q_{i k} \nabla_{j} Q_{j k}-L_{3} \varepsilon_{i j k} Q_{i l} \nabla_{k} Q_{j l} \\
& +\frac{1}{2 \chi_{0}} \mathbf{P}_{\mathbf{m}}{ }^{2}+G Q_{i j} P_{m} i P_{m} j-P_{m} i E_{i}
\end{aligned}
$$

where $F_{0}$ is the free energy of the isotropic phase. $A=a\left(T-T^{*}(P)\right)$ and $T^{*}(P)$ is the critical temperature for a hypothetical second order transition. All other coefficients, as well as $a$ and $\alpha_{0}$ 
are assumed to be temperature independent. $\chi_{0}$ is the polarizability of the isotropic phase. The parameter $G$ represents the anisotropy of the polarizability in the cholesteric phase. $\gamma$ may be positive or negative. The term $\sim P_{k} P_{n} Q_{k l} Q_{n l}$ is neglected for the simplicity. $L_{1}, L_{2}$ and $L_{3}$ are the orientational elastic constants. $\varepsilon_{i j k}$ is the Levi-Cevita antisymmetric tensor of the third rank. The term proportional to $L_{3}$ violates parity and is responsible for the formation of a helical ground state. We assume $L_{3}>0$. It must be stressed that in this manuscript we consider only the uniaxial case and also neglect the blue phases for simplicity.

In accordance with the experimental phase diagram (shown in Fig.1), $T^{*}(P)$ can be expanded as

$$
T^{*}(P)=T_{0}+u P
$$

where $u$ is a positive constant.

Now we consider the phases in which the nematic order is spatially homogeneous, i.e. $S=$ const. Equation (2.1) can be simplified if one assumes that the polarization is aligned along the nematic director $\mathbf{n}$ i.e. $\mathbf{P}_{\mathbf{m}}=\left(0,0, P_{m}\right)$. We choose $\mathbf{E}=(0,0, E)$. The substitution of $Q_{i j}$, and $\mathbf{P}_{\mathbf{m}}$ in Eq. (2.1) leads to the free energy

$$
\begin{aligned}
F= & F_{0}+\frac{3}{4} A S^{2}-\frac{1}{4} B S^{3}+\frac{9}{16} C S^{4}+\frac{1}{2 \chi_{0}} P_{m}^{2}-\frac{1}{2} G P_{m}^{2} S \\
& +\frac{9}{4} L S^{2} k_{0}^{2}-\frac{9}{4} L_{3} S^{2} k_{0}-P_{m} E
\end{aligned}
$$

where $C=\left(C_{1}+C_{2} / 2\right)$ and $L_{1}=-2 L_{2} / 3 \equiv L$.

After minimizing the free energy (2.3) with respect to $k_{0}$, the wave vector of the helix in the cholesteric phase can expressed as

$$
k_{0}=\frac{L_{3}}{2 L_{1}}
$$

Equation (2.4) shows that $L_{3}>0$ is required in order to obtain a positive value of $k_{0}$ in the cholesteric phase. However, the wave vector $k_{0}$ of the cholesteric phase may be positive or negative, since there are two possible ways of rotation of the cholesteric helix. Minimizing the free energy (2.3) with respect to the polarization $P_{m}$ leads to:

$$
P_{m}=E \chi_{0} M
$$

where $M=\left(1-G \chi_{0} S\right)^{-1}$.

The substitution of $k_{0}$ and $P_{m}$ from Eqs. (2.4)-(2.5) into Eq. (2.3), we obtain 


$$
F=F_{0}^{*}+\frac{3}{4} A^{*} S^{2}-\frac{1}{4} B S^{3}+\frac{9}{16} C S^{4}-\frac{1}{2} \gamma \chi_{0}^{2} E^{2} S-\frac{1}{2} G^{2} \chi_{0}^{3} E^{2}-\frac{1}{2} G^{3} \chi_{0}^{4} E^{2} S^{3}
$$

The renormalized coefficients are

$$
\begin{aligned}
& F_{0}^{*}=F_{0}-\frac{E^{2} \chi_{0}}{2}, \\
& A^{*}=A-\frac{3 L_{3}^{2}}{4 L} .
\end{aligned}
$$

First we consider the I-Ch transition in the absence of external electric field. The conditions for the first order I-Ch transition can be obtained as

$$
F(S)=0, F^{\prime}(S)=0, F^{\prime \prime}(S) \geq 0
$$

The temperature and pressure dependence of $S$ can be expressed as

$$
\left(S-S^{+}\right)^{2}=S^{+2}-\frac{a}{3 C}\left[T-u p-T_{0}-\left(3 L_{3}^{2} / 4 L a\right)\right]
$$

Thus $S$ changes with the change of pressure in the cholesteric phase. When $S$ is fixed, Eq. (2.8) may be rewritten as

$$
T(P)=T_{10}(S)+u P
$$

where

$$
T_{10}(S)=T_{0}+\left(3 L_{3}^{2} / 2 L a\right)-(3 C / 2 a) S\left(S-S^{+}\right)
$$

where $S^{+}=B / 6 C$. However a change on $S$, Eq. (2.8) shows the nonlinearity of $T$ vs. $P$. The jump of the orientational order parameter at the I-Ch transition is given by

$$
S_{I-C h}=\frac{2 B}{9 C}
$$

The I-Ch transition temperature is given by

$$
T_{I-C h}=T_{0}+\frac{B^{2}}{27 a C}+\frac{3 L_{3}^{2}}{4 L a}+u P
$$

The analysis of Eq. (2.6) shows the influence of the external electric field on the cholesteric liquid crystal results in two main effects. First, the electric field produces a shift of the transition temperature $T_{I-C h}^{*}$ which is proportional to the square of the electric field

$$
\Delta T_{I-C h}^{*}(P)=m E^{2}
$$


with $m=\frac{G^{2} \chi_{0}^{2}}{3 a}$. Secondly, the external electric field induces weak orientational ordering in the isotropic phase. The orientational order parameter induced by an electric field in the isotropic phase is calculated to a first approximation $(B=0$ and $C=0)$ and can be expressed as

$$
S(E)=\frac{U}{\left(T-T_{0}-\frac{3 L_{3}^{2}}{4 L a}-u P\right)} E^{2}+\frac{V}{\left(T-T_{0}-\frac{3 L_{3}^{2}}{4 L a}-u P\right)^{2}} E^{4}
$$

where

$$
\begin{aligned}
& U=\frac{G \chi_{0}^{2}}{3 a}, \\
& V=\frac{2 G^{3} \chi_{0}^{5}}{9 a^{3}} .
\end{aligned}
$$

Note that in the first approximation, $S(E)=\frac{U}{\left(T-T_{0}-\frac{3 L_{3}^{2}}{4 L a}-u P\right)} E^{2}$.

We will now calculate the frequency dependent $N D E$ in the isotropic phase of the I-Ch transition. The equation of motion, which we will write down as torque balance equation, can now be written

$$
-\frac{\partial F}{\partial S}-\Gamma \frac{d S}{d t}=0
$$

where $\Gamma$ is the rotational viscosity. Introducing a time dependence of the order parameter $S$ and the electric field $E$ as $S=S_{0} e^{j \omega t}$ and $E=E_{0} e^{j \omega t}$, Eq. (2.15) to a first approximation ( $B=0$ and $C=0)$ give

$$
-\frac{3}{2} A^{*} S+\frac{1}{2} G \chi_{0}^{2} E^{2}-j \omega \Gamma=0
$$

Solving Eq. (2.16), we get the frequency dependent induced order parameter in the isotropic phase as

$$
S(\omega)=\frac{U}{\left(T-T_{0}-\frac{3 L_{3}^{2}}{4 L a}-u P+j \omega \frac{2 \Gamma}{3}\right)} E^{2}
$$

The $N D E$ denotes the change in the dielectric permittivity of a material that originates from the application of strong static electric field $\mathbf{E}$. The $N D E$ is widely analogous to the electro-optic Kerr effect which applies to the case of optical frequencies. An anisotropy property is proportional to the induced order. Hence the dielectric permittivity in the isotropic phase can be expressed as

$$
\Delta \varepsilon(E)=\varepsilon(E)-\varepsilon(0)=\left(\Delta \varepsilon_{f}\right)_{\max } S(E, \omega)
$$

where $\varepsilon(E)$ and $\varepsilon(0)$ are the dielectric permittivities in a strong $(E)$ and weak (measuring) electric field. $\left(\Delta \varepsilon_{f}\right)_{\max }$ denotes the maximum anisotropy of the dielectric permittivity for the given frequency $f$. Combining Eqs. (2.17) and (2.18) we find

$$
\varepsilon_{N D E}=\frac{\varepsilon(E)-\varepsilon(0)}{E^{2}}=\varepsilon_{N D E}^{R e}-j \varepsilon_{N D E}^{I m}
$$


where

$$
\begin{gathered}
\varepsilon_{N D E}^{R e}=\frac{W}{\left(T-T_{0}^{*}\right)+\frac{4 \omega^{2} \Gamma^{2}}{9 a^{2}\left(T-T_{0}^{*}\right)}} \\
\varepsilon_{N D E}^{I m}=\frac{2 \omega \Gamma G \chi_{0}^{2}}{9 a^{2}\left[\left(T-T_{0}^{*}\right)^{2}+\frac{4 \omega^{2} \Gamma^{2}}{9 a^{2}}\right]}
\end{gathered}
$$

where $W=\left(\Delta \varepsilon_{f}\right)_{\max } U$ and $T_{0}^{*}=T_{0}+\frac{3 L_{3}^{2}}{4 L a}+u P$.

To parameterize the pretransitional anomaly in $5^{*} \mathrm{CB}$, Rzoska et al [15] measured the apparent amplitude $A_{N D E}(T)$ of the $N D E$. From the relation (2.20), the apparent amplitude can be expressed as

$$
A_{N D E}(T)=N D E \times\left(T-T_{0}^{*}\right)=W-\frac{4 W \omega^{2} \Gamma^{2}}{9 a^{2}\left(T-T_{0}^{*}\right)^{2}}
$$

From an alternative point of view, we can take $A=a\left(P^{*}-P\right) . P^{*}$ is supercooling pressure. In this case $\varepsilon_{N D E}$ can be expressed as

$$
\varepsilon_{N D E}=\frac{\varepsilon(E)-\varepsilon(0)}{E^{2}}=\varepsilon_{N D E}^{R e}-j \varepsilon_{N D E}^{I m}
$$

where

$$
\begin{gathered}
\varepsilon_{N D E}^{R e}=\frac{W}{\left(P^{*}-\frac{3 L_{3}^{2}}{4 L a}-P\right)+\frac{4 \omega^{2} \Gamma^{2}}{9 a^{2}\left(P^{*}-\frac{3 L_{3}^{2}}{4 L a}-P\right)}} \\
\varepsilon_{N D E}^{I m}=\frac{2 \omega \Gamma G \chi_{0}^{2}}{9 a^{2}\left[\left(P^{*}-\frac{3 L_{3}^{2}}{4 L a}-P\right)^{2}+\frac{4 \omega^{2} \Gamma^{2}}{9 a^{2}}\right]}
\end{gathered}
$$

\section{COMPARISON WITH EXPERIMENT}

In this section we will compare our theoretical results with the available experimental results. According to Eq. (2.12), the I-Ch transition temperature $T_{I-C h}$ increases with increasing pressure. The pressure dependence of the I-Ch transition temperature of $5^{*} \mathrm{CB}$ was reported by Rzoska et al [14]. The experimental phase diagram for $T_{I-C h}$ vs $P$ is a straight line. From Eq. (2.9), when $S$ is fixed, $T$ vs $P$ should be a straight line. Equation (2.12) is fitted with our measured data of $T_{I-C h}$ as a function of pressure for $5^{*} \mathrm{CB}$ taking $\left(T_{0}+\frac{B^{2}}{27 a C}-\frac{3 L_{3}^{2}}{4 L a}\right)$ and $u$ as fit parameters. The fit (solid) line and the measured data (solid circles) are shown in Fig. 1. The fit yields $\left(T_{0}+\frac{B^{2}}{27 a C}-\frac{3 L_{3}^{2}}{4 L a}\right)=250 K$ and $u=0.364 K / M P a$. The temperature dependence of the real part 
of the $N D E$ for a fixed $P$ given by Eq. (2.19) can easily be verified with Fig. 6 of Rzoska et al [15]. As for the pressure and temperature dependence the mean-field value of the critical exponent $\varepsilon_{N D E}^{R e} \propto\left(P^{*}-\frac{3 L_{3}^{2}}{4 L a}-P\right)^{-\gamma} \propto\left(T-T_{0}^{*}\right)^{-\gamma}$ gives $\gamma=1$ which agrees with experimental observations. Furthermore, to check Eq. (2.20), $\varepsilon_{N D E}^{-1}$ vs $T-T_{0}^{*}$ of Rzoska et al [15] for constant pressure is plotted in Fig.2. The form of Eq. (2.20) shows that there are several unknown parameters. It is unphysical to take all these parameters as fit parameters. We have, therefore, fitted Eq. (2.20) with the measured $\varepsilon_{N D E}^{R e}(T)$ data using $W, \Gamma$ and $\frac{4}{9 a^{2}}$ as fit parameters. The solid line in Fig. 2 is the best fit of the real part of Eq. (2.19) for the different frequencies. The values obtained for the fit parameters are listed in Table I. The temperature dependence of the apparent amplitude of the $N D E$ in the isotropic phase for a fixed $P$ is shown in Fig. 3. The line is the fit to the theoretical expression Eq. (2.22) using $W, \frac{4}{9 a^{2}}$ and $\Gamma$ as fit parameters. The values obtained for the fit parameters are $W=230.73 \times 10^{-16} \mathrm{~m}^{2} V^{-2} \mathrm{~K}, \frac{4}{9 a^{2}}=0.92$ $K^{2} k H z^{-2}\left(M H z^{-2}\right) P_{o i s e}{ }^{-2}$ and $\Gamma=0.82$ Poise. The fit to the measured values are good in Fig. 1-Fig. 3. Table I shows that the rotational viscosity $\Gamma$ decreases with the increase of frequency as expected. This behavior clearly support the theoretical analysis. To the best of the authors knowledge there are still no experimental estimations of the frequency dependent rotational viscosity.

\section{CONCLUSION}

We have examined the pressure effect on the I-Ch transition in the mean-field description. The present analysis provides the first theoretical support to the experimental observations of the $N D E$ in the isotropic phase of the I-Ch transition [13 15]. The effect of pressure on the I-Ch transition is to increase the transition temperature with pressure. The I-Ch transition is found to be a first order even at high pressure. Our theory explain the unusual positive and negative pretransitional effect in the isotropic phase of the I-Ch transition. The same pretransitional phenomena is observed in the isotropic phase of the I-Ch transition similar to the I-N and I-SmA transitions. The critical exponent $\gamma=1$ indicate the fluid like analogy in the isotropic phase of the I-Ch transition.

\section{ACKNOWLEDGMENTS}

PKM thanks the Alexander von Humboldt Foundation for equipment and book grant. SC thanks the KVPY for Fellowship. SJR was supported by Ministry of Science and Education 
(Poland) Grant No. 202231737. 
[1] P. G. de Gennes, Mol. Cryst. Liq. Cryst.12 (1971) 193.

[2] J. Cheng, R. B. Meyer, Phys. Rev. A 9 (1974) 2744.

[3] C. C. Yang, Phys. Rev. Lett. 28 (1972) 955.

[4] D. Coates, G. W. Gray, Phys. Lett. A 51 (1975) 335.

[5] T. Harada, P. P. Crooker, Phys. Rev. Lett. 34 (1975) 1259.

[6] E. I. Kats, Sov. Phys. JETP 38 (1974) 1242.

[7] A. Brazovskii, S. G. Dmitriev, Sov. Phys. JETP 42 (1975) 497.

[8] A. Brazovskii, Sov. Phys. JETP 41 (1975) 85.

[9] A. Brazovskii, V. M. Filev, Sov. Phys. JETP 48 (1978) 573.

[10] L. Longa, D. Monselesan, H. R. Trebin, Liq. Cryst. 5 (1989) 889.

[11] K. Muralidhar, M. L. S. Swamy, S. N. Rao, K. S. Rao, Liq. Cryst. 9 (1991) 583.

[12] R. Seidin, D. Mukamel, D. W. Allender, Phys. Rev. E 56 (1997) 1773.

[13] S. J. Rzoska, A. Drozd-Rzoska, M. Gorny, J. Jadzyn, J. Ziolo, K. Czuprynski, R. Dabrowski, J. Non. Cryst. Solid 307-310 (2002) 311.

[14] S. J. Rzoska, M. Paluch, S. Pawlus, A. Drozd-Rzoska, J. Ziolo, J. Jadzyn, K. Czuprynski, R. Dabrowski, Phys. Rev. E 68 (2003) 031705.

[15] A. Drozd-Rzoska, S. J. Rzoska, M. Paluch, S. Pawlus, J. Ziolo, P. G. Santangelo, C. M. Ronald, K. Czuprynski, R. Dabrowski, Phys. Rev. E 71 (2005) 011508.

[16] P. K. Mukherjee, J. Phys.: Condens. Matter 10 (1998) 9191.

[17] A. Drozd-Rzoska, S. J. Rzoska, J. Ziolo, Phys. Rev. E 55 (1997) 2888.

[18] A. Drozd-Rzoska, S. J. Rzoska, J. Ziolo, Phys. Rev. E 61 (2000) 5349.

[19] A. Drozd-Rzoska, S. J. Rzoska, J. K. Czuprynski, Phys. Rev. E 61 (2000) 5355.

[20] A. Drozd-Rzoska, S. J. Rzoska, J. Ziolo, J. Jadzyn, Phys. Rev. E 63 (2001) 052701.

[21] P. G. de Gennes, J. Prost, The Physics of Liquid Crystals (Clarendon Press, Oxford, 1993). 
TABLE I: Values of the observed frequencies and the corresponding various fitted parameters in the isotropic phase of the I-Ch transition in $5^{*} \mathrm{CB}$, as derived from a fit of Eq. (2.20) to the measured data of Ref. [15].

\begin{tabular}{lccc}
\hline$\omega$ & $W$ & $\frac{4}{9 a^{2}}$ & $\Gamma$ \\
& $\left(10^{-16} \mathrm{~m}^{2} V^{-2} K\right)$ & $\left(K^{2} k H z^{-2}\left(\mathrm{MHz}^{-2}\right)\right.$ Poise $\left.^{-2}\right)$ & $($ Poise $)$ \\
\hline $66 k H z$ & 233.99 & 1.29 & 1.13 \\
$3.2 \mathrm{MHz}$ & -123.86 & 1.29 & 0.96 \\
$6.6 \mathrm{MHz}$ & -151.84 & 1.29 & 0.86 \\
\hline
\end{tabular}




\section{Figure Captions:}

FIG. 1: The pressure dependence of the I-Ch transition temperature. The solid line is the best fit of Eq. (2.12).

FIG. 2: The temperature dependence of the $N D E$ in the isotropic phase of $5^{*} \mathrm{CB}$. The measured data are from Ref. [15] and the line is the best fit of Eq. (2.20).

FIG. 3: The temperature dependence of the apparent amplitude $A_{N D E}$ of the $N D E$ in the isotropic phase of $5^{*} \mathrm{CB}$. The measured data are from Ref. [15] and the line is the best fit of Eq. (2.22). 
FIG.1

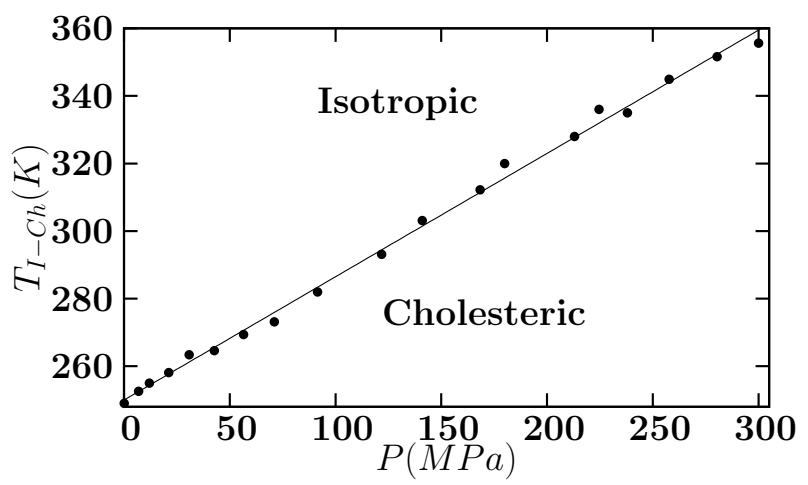


FIG.2

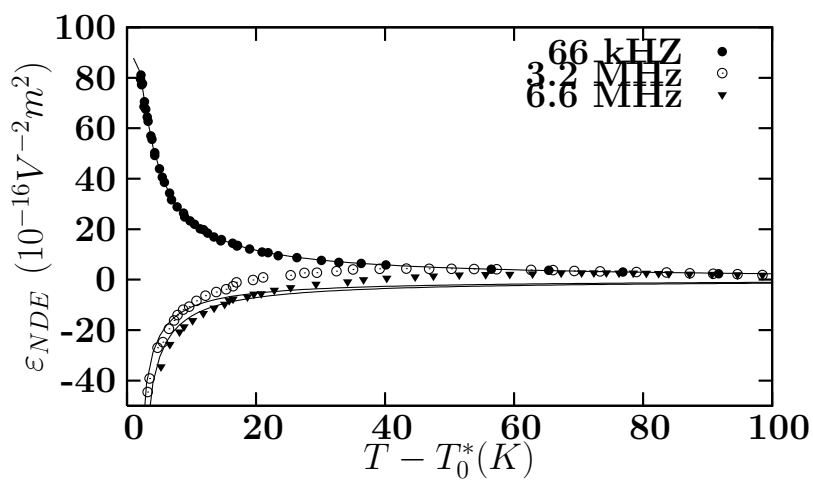


FIG3.

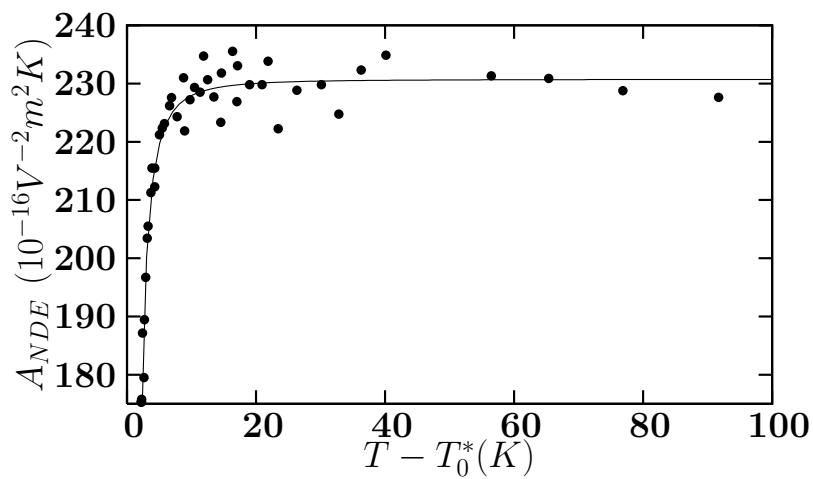

